\title{
Formulation of an Emulgel Containing Strawberry Fruit Extract and in-vivo Evaluation for Different Skin Parameter
}

\author{
Rizwana Kausar*, Naveed Akhtar \\ Department of Pharmacy, Faculty of Pharmacy and Alternative Medicine, Thelslamia University of Bahawalpur, 63100, PAKISTAN.
}

\begin{abstract}
Introduction: It has been indicated from previous research that strawberry consumption may be beneficial to improve skin appearance and its extract has antioxidant and antiinflammatory effects. Objective: The aim of present study was to fabricate an emulgel containing extract of strawberry (4\%) and in-vivo evaluation on different skin parameters versus its base taken as control. Methods: Strawberry fruit extract, which was obtained by concentrating methanolic extract of strawberry fruit, was entrapped in the inner oily phase of $(\mathrm{o} / \mathrm{w})$ emulsion and then incorporated this emulsion into gel to develop emulgel. Both base (without extract) and active formulation were stored at different accelerated conditions for a period of six weeks to predict their stability. For in-vivo evaluation, both formulations were applied to the cheeks of healthy female human volunteers $(n=13)$ for twelve weeks and various skin parameters were evaluated to measure any effect on skin produced by base and active formulations. The instrumental measurements were carried out with controlled temperature $\left(18-20.6 \mathrm{C}^{\circ}\right)$ and relative humidity (55$65 \%$ ). Results: Skin melanin, erythema and sebum showed a significant (decrease by active formulation. A significant (increase in skin moisture content was found by active formulation.Conclusion: Strawberry emulgel can be used to improve texture of skin and enhance whiting effect without causing any skin irritation.

Key words: Strawberry, Emulgel, Stability, Melanin, Sebum, Erythema.
\end{abstract}

\section{INTRODUCTION}

The skin is the largest organ system of the body, both in weight and surface area that covers the external surface of the body. Skin have large no of characteristics such as texture, color and thickness varies with location, age and sex. Skin stores water, fat and vitamin $\mathrm{D}$ and regulates the body temperature. The skin is divided into three layers known as the epidermis, dermis and the subcutaneoustissue. ${ }^{1}$ Skin is affected by various intrinsic and extrinsic factors. These factors cause changes in the texture of skin. ${ }^{2}$ When emulsions and gels are used in combined form the dosage forms are referred as Emulgels. Actually, the presence of a gelling agent converts a classical emulsion into an emulgel. Emulgels for dermatological use have several favorable properties such as being greaseless, thixotropic, easily removable, easily spreadable, non-staining, emollient, water soluble, longer shelf life, bio-friendly, pleasing appearance and transparent. ${ }^{3}$ Additional values can be given to emulgels by incorporating plant or fruit extractswith specific cosmeceutical effects.

Most of the active constituents are isolated from natural herbs are used in cosmetics as potential antioxidants. ${ }^{4}$ Strawberry, belonging to the family Rosaceae is a fruit that has a rich history of traditional and pharmacological uses. It has significant amount of phenolic compounds, vitamin $\mathrm{C}$, anthocyanins such as pelargonidin-3-glucoside (pel-glc), carotenoids and flavonoids. ${ }^{5}$ Strawberryfruit and leaf extract is traditionally used as an anti-inflammatory, antioxidant, astringent,
Submission Date: 24-01-2017; Revision Date: 10-04-2017; Accepted Date: 10-05-2017

DOI: 10.5530/ijper.51.4.100 Correspondence: Rizwana Kausar, Department of Pharmacy, Faculty of Pharmacy and Alternative Medicine, Thelslamia University of Bahawalpur, 63100, PAKISTAN.

Contact no.: 0092345106247 E-mail: rizvi_awan@yahoo. com

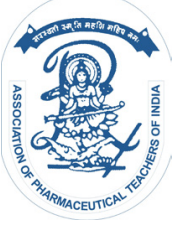

www.ijper.org 
anti-apoptosis and diuretic. ${ }^{6}$ Previous literature indicates that no stable and suitable skin formulations of strawberryfruit is yet been developed. The aim of this investigational study was to develop an emulgel containing strawberry fruit extract and evaluate its effect on various physiologic functions of skin like melanin, erythema, moisture content and skin sebum.

\section{MATERIALS AND METHODS}

\section{Materials}

Methanolic extract of strawberry fruit and distilled water were prepared in the laboratory of Department of Pharmacy, The Islamia University of Bahawalpur, Pakistan. Propylene glycol, triethnolamine, liquid paraffin and methanol were obtained from Merck (Germany), span 80, tween 20, methyl paraben and carbopol 940 were purchased from Sigma, USA.

\section{Apparatus}

Electrical balance (Precisa BJ-210, Switzerland), Rotary evaporator (Eyela, Co. Ltd. Japan), refrigerator (Dawlance, Pakistan), pH-meter (WTW pH-197i, Germany), Mexameter ${ }^{\circledR}$ (MPA-5) (Courage + Khazaka, Germany), Corneometer ${ }^{\circledR}$ MPA-5 (Courage + Khazaka, Germany), Sebumeter ${ }^{\circledR}$ MPA-5 (Courage + Khazaka, Germany), Elastometer ${ }^{\circledR}$ EM 25 (Courage + Khazaka, Germany), homogenizer (Euro-Star, IKA D 230, Germany)and UV spectrophotometer-16 (Shimadzo Japan).

\section{Plant material and identification}

Strawberryfruit was purchased from the local market of Sargodha, Pakistan. Identification of the fruit was made at the Botany Department of University of Sargodha, Pakistan. The specimen (Voucher\# 6027) was deposited in the Laboratory of Biosystematics \& Medicinal Plants, Department of Botany, University of Sargodha, Pakistan, for future research reference.

\section{Method}

In this study, base and active formulation were divided into four samples and these samples were kept at $8^{\circ} \mathrm{C}$ in a refrigerator, at $25^{\circ} \mathrm{C}, 40^{\circ} \mathrm{C}$ and $40^{\circ} \mathrm{C}$ with $75 \% \mathrm{RH}$ (relative humidity) in stability chambers. These were observed organoleptically with respect to change in color, phase separation and liquefaction for a period of 60 days at specific time intervals. After the development of stable emulgel in-vivo characterization was carried out to evaluate this formulation for different skin parameters.

\section{Preparation of emulgel}

An emulgel (base as well as active formulation) was developed by the addition of $\mathrm{o} / \mathrm{w}$ emulsion to the gelling agent. Aqueous phase (tween 20, propylene glycol, methyl paraben and distilled water) of emulsion was prepared by heating to $75 \pm 1^{\circ} \mathrm{C}$. Similarly, oily phase (paraffin oil and span 80) was heated to the same temperature. Strawberry fruit extract was added to aqueous phase. Then oily phase was added drop wise to the aqueous phase with continued stirring until complete oily phase was added to prepare $\mathrm{o} / \mathrm{w}$ emulsion. Carbopol 940 ( $2 \mathrm{~g})$ was dispersed in water with q.s. to $100 \mathrm{ml}$. After complete dispersion, triethnolamine was added drop wise, and after each addition $\mathrm{pH}$ was checked until it reached within the range of skin $\mathrm{pH}$ (4.5-6.5). Finally, prepared o/w emulsion was added gradually in prepared gel under continuous stirring at $2000 \mathrm{rpm}$ by homogenizer for $15 \mathrm{~min}$. Then, the speed was reduced to $1000 \mathrm{rpm}$ for $5 \mathrm{~min}$, and then the speed further was decreased to $500 \mathrm{rpm}$ for a period of $5 \mathrm{~min}$ for proper homogenization until the emulsion cooled at room temperature.During this homogenizing time, 2-3 drops of fragrance (fruit oil) were added to give pleasant fragrance to the emulgel.

\section{Study Protocol}

13 healthy human female volunteers were selected whose ages were between 25 and 30 years. Prior to the dermatological tests, a dermatologist examined the volunteers for any skin disorder especially on cheeks. Each volunteer was provided with two formulations. One formulation was the base and the other formulation was active formulation containing $4 \%$ strawberry fruit extract. Each formulation was marked with "left" or "right" indicating the application of base and active formulation respectively. Each volunteer was instructed to apply $5 \mathrm{mg}$ of both base as well as active formulation twice a day for the period of 3 months. Each volunteer was instructed to come at $2^{\text {nd }}$ week, $4^{\text {th }}, 8^{\text {th }}, 10^{\text {th }}$ and $12^{\text {th }}$ week of investigational period for the dermatological tests.

\section{Ethical approval}

This study was approved by the Pharmacy Research Ethics Committee (PREC) for in-vivo studies(Ref. No.662015/PREC),The Islamia University of Bahawalpur, Pakistan and was directed according to the international guidelines of Helsinki Declaration.

\section{Statistical and mathematical analysis}

The percentage changes in the individual values of different skin parameters were determined by the following formula; 


$$
\text { Percentage change }(\%)=\left[\frac{(\mathrm{A}-\mathrm{B})}{\mathrm{B}}\right] \times 100
$$

Where, $A=$ individual value on any parameter at $2^{\text {nd }}$, $4^{\text {th }}, 8^{\text {th }}, 10^{\text {th }}$ and $12^{\text {th }}$ week. $B=$ zero hour value of that parameter.

Obtained values for various skin parameters (melanin, erythema, skin moisture, skin sebum and elasticity) were analyzed statistically using SPSS 20.0. Two -way ANOVA at 5\% level of significance (for variation between different time intervals) and paired sample $\mathrm{t}$-test (the variation between the two formulations) were used for statistical analysis.

\section{RESULTS AND DISCUSSION}

\section{Stability studies}

\section{Color}

Freshly prepared base and active formulation were white and light pink respectively. There was no change in color of base active formulation under various storage conditions up to the end of investigational period of 60 days. This may be attributed to the presence of paraffin oil which is a transparent, colorless and is a mixture of hydrocarbons, ${ }^{7}$ and the active ingredient i.e. polyphenols contained in strawberry extract. Polyphenols are well recognized to have bactericidal activity. ${ }^{8}$ This indicates that both base and active formulation were stable up to study period of 60 days.

\section{Phase separation}

The samples of base were stable at $8^{\circ} \mathrm{C}, 25^{\circ} \mathrm{C}$ and $40^{\circ} \mathrm{C}$ but a light phase separation was observed at $40^{\circ} \mathrm{C}+75 \%$ $\mathrm{RH}$ on $60^{\text {th }}$ day of investigation period this may be attributed to the storage conditions. The active formulation was stable at $8^{\circ} \mathrm{C}, 25^{\circ} \mathrm{C}$ and $40^{\circ} \mathrm{C}$ and $40^{\circ} \mathrm{C}+75 \%$ $\mathrm{RH}$ throughout the study period of 60 days.

\section{Liquefaction}

No liquefaction was found in the samples of base and active formulation kept at $8^{\circ} \mathrm{C}, 25^{\circ} \mathrm{C}$ throughout the observation period. A minor liquefaction was found in the samples of active formulation and base kept at $40^{\circ} \mathrm{C}$ and $40^{\circ} \mathrm{C}+75 \% \mathrm{RH}$ on the $52^{\text {nd }}$ and $60^{\text {th }}$ day of study period. In the previous literature it has revealed that according to stoke's law, the rate of creaming is inversely proportional to the viscosity of continuous phase. So the viscosity of base and active formulation reduces as the creaming increases with increasing temperature causing liquefaction. ${ }^{?}$

\section{pH test}

The $\mathrm{pH}$ of freshly prepared base and active formulation was 6.20 and 5.82 respectively, which is considered within the range of skin $\mathrm{pH}$. The human skin $\mathrm{pH}$ ranges from 4.5 to $6.5 .^{10}$ The $\mathrm{pH}$ of the samples of base and active formulation kept at different storage temperatures was found to be gradually decreasing but within the skin $\mathrm{pH}$ range. This reduction in $\mathrm{pH}$ might be due to the oxidation process and presence of acidic constituents in extract as plant extracts were acidic in nature. By applying two-way ANOVA, it was depicted that the change in $\mathrm{pH}$ of samples of base and active formulation was significant at different conditions of time and temperature. The average $\mathrm{pH}$ values of samples kept at $8^{\circ} \mathrm{C}, 25^{\circ} \mathrm{C}$, $40^{\circ} \mathrm{C}$ and $40^{\circ} \mathrm{C}$ with $75 \% \mathrm{RH}$ are represented in Table 1.

\section{In-vivo characterization}

\section{Skin melanin}

In this investigational study, the effect of the base and active formulation on skin melanin content was measured by using non-invasive probe Mexameter. The melanin content was measured for 12 weeks of study period at different time intervals for each volunteer. It was depicted that base increased the melanin content irregularly till the end of $12^{\text {th }}$ week while active formulation gradually decreased throughout the study period, as given in Figure 1. With the help of two- way ANOVA, it was observed that active formulation produced significant difference in average $\%$ age changes of skin melanin values with respect to time; whereas the Base produced insignificant effects with time. With the help of paired sample t-test, significant differences were observed between the melanin effects of Base and active formulation during the whole study period. It was concluded that the decreased melanin content after application of active formulation may be related to antioxidant activity

\begin{tabular}{|c|c|c|c|c|c|}
\hline \multicolumn{6}{|c|}{ Values of $\mathrm{pH}$ (mean \pm SEM) } \\
\hline \multicolumn{6}{|c|}{ Storage condition } \\
\hline Formulation & Fresh & $8^{\circ} \mathrm{C}$ & $25^{\circ} \mathrm{C}$ & $40^{\circ} \mathrm{C}$ & $40^{\circ} \mathrm{C}+75 \% \mathrm{RH}$ \\
\hline Base & $6.20 \pm 0.018$ & $6.00 \pm 0.136$ & $5.93 \pm 0.017$ & $5.88 \pm 0.046$ & $5.83 \pm 0.011$ \\
\hline Active & $5.82 \pm 0.019$ & $5.80 \pm 0.017$ & $5.75 \pm 0.028$ & $5.70 \pm 0.017$ & $5.62 \pm 0.023$ \\
\hline
\end{tabular}


due to the presence of flavonoids. ${ }^{11}$ The antioxidant activity of phenolics is due to their redox action. They act as hydrogen donors, free radical scavengers, and metal chelators.

\section{Erythema}

In this study, the irritation was evaluated by Mexameter after every two weeks for the base and active formulation throughout the study period of 12 weeks. It was depicted that erythema contents increased irregularly throughout the study period, whereas pronounced decrease in erythema contents was found after the application of active formulation as shown in Figure 2. With the help oftwo- way ANOVA, it was observed that active formulation produced significant difference in average $\%$ age changes of skin melanin values with respect to time; whereas the Base produced insignificant effects with time. With the help of paired sample t-test, significant differences were observed between the melanin effects of Base and active formulation during the whole study period. It was concluded from our findings that the base increased while active formulation gradually decreased erythema contents throughout the study period. So the active formulation containing $4 \%$ blackberry fruit extract can be used safely without any skin irritation.

\section{Skin moisture content}

After the application of base there was a slight increase in moisture content and it was investigated by corneometer at $2^{\text {nd }}, 4^{\text {th }}, 6^{\text {th }}, 8^{\text {th }}, 10^{\text {th }}$ and $12^{\text {th }}$ week of study period; whereas a pronounced increase in moisture content was observed after the application of active formulation throughout the study time of 12 weeks as shown in Figure 3. After applying the statistical analysis (two-way ANOVA test) it was found that E1 as well as base produced significant increase in moisture content with respect to the time. With the help of paired sample t-test analysis, it was evident that significant differences in the skin moisture values were observed by E1 and its respective base after the study period of 12 weeks. This significant increase in moisture content may be linked to the presence of vitamin $\mathrm{C}$ in the fruit extract. ${ }^{5}$ Vitamin $\mathrm{C}$ can improve skin hydration level by activating collagen synthesis.

\section{Skin sebum}

Skin sebum content was measured by sebumeter. It was found that after the application of active formulation, a pronounced decrease in sebum values was observed throughout the study period of 12 weeks, whereas respective base caused a gradual increase in sebum content during the whole study period of 12 weeks as

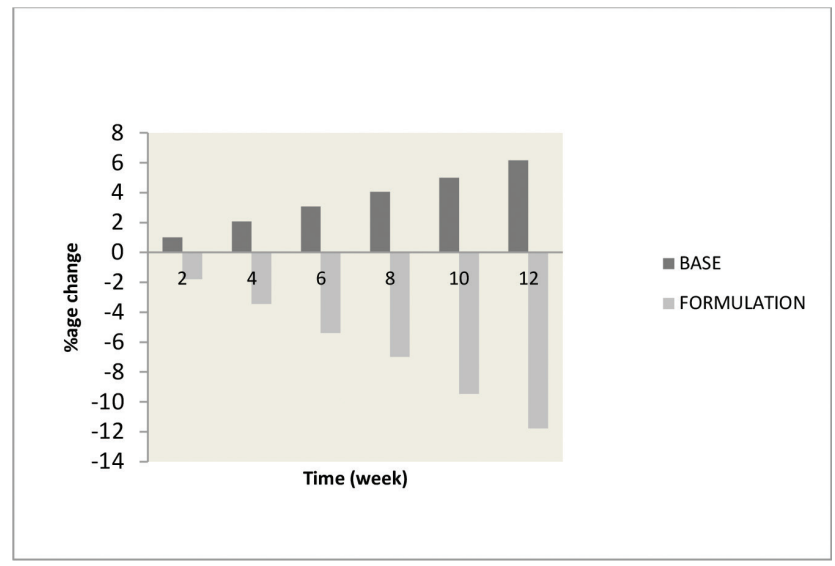

Figure 1: Percentage of change in skin melanin after the application of active formulation and base.



Figure 2: Percentage of change in skin erythema after the application of active formulation and base.

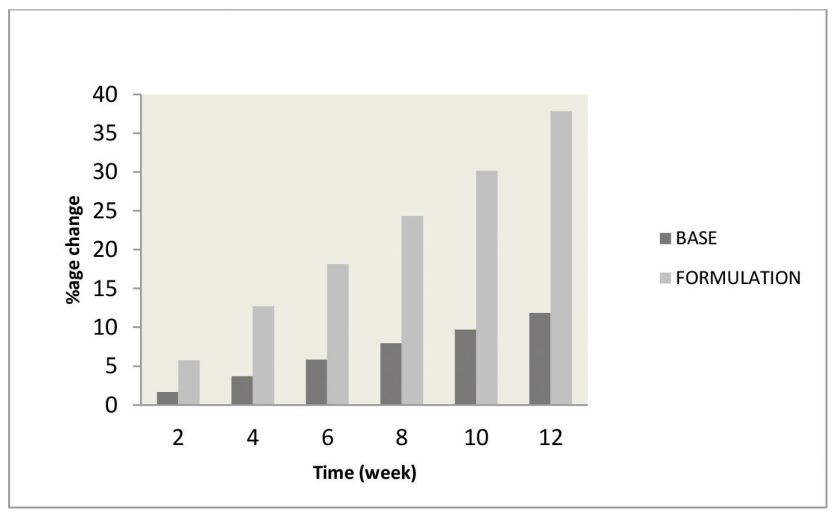

Figure 3: Percentage of change in skin moisture content after the application of active formulation and base.

shown in Figure 4. With the help of two-way analysis of variance (ANOVA) test, it was assessed that active formulation statistically decreased the sebum values after 12 weeks of investigational period while its respective Base produced insignificant results after 12 weeks of study period. When paired sample t-test was applied on sebum values of active formulation and base, statistically significant difference was found after 12 weeks of study period. Reduction in sebum contents after application of active formulation may be attributed to the presence 


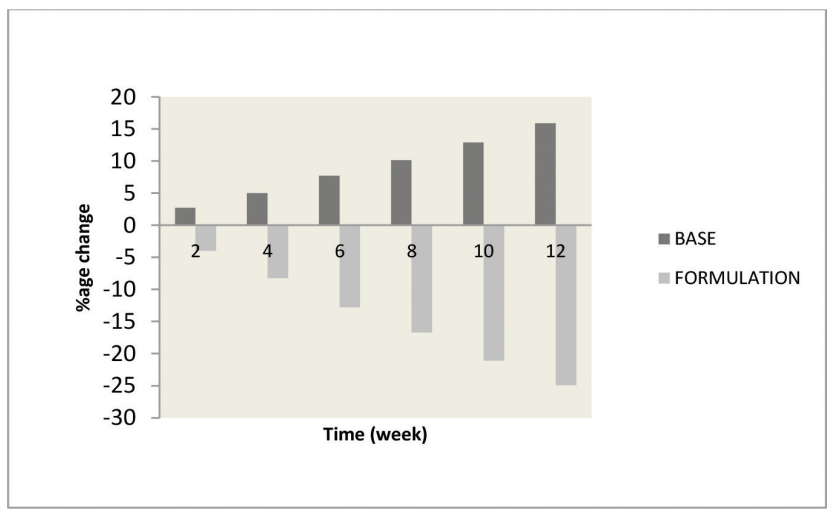

Figure 4: Percentage of change in skin sebum content after the application of active formulation and base.

of polyphenol enriched fruit extract. It has narrated that $\alpha$-reductase type- 1 enzyme may cause the enlargement of sebaceous glands; by converting testosterone to dihydro-testosterone. So enlarged sebaceous glands secret high sebum contents in skin. Various phytochemical constituents such as sterols and polyphenols may prevent the sebum production by inhibiting $\alpha$-reductase type-1 enzyme. ${ }^{12}$

\section{CONCLUSION}

It can be concluded that a stable emulgel containing extract of Strawberry fruit $(4 \%)$ can be developed and used to decrease skin melanin and sebum content and to increase skin hydration level without causing any skin irritation. Furthermore the strawberry emulgel can also be used as cost effective skin whiting treatment.

\section{ACKNOWLEDGEMENT}

The authors wish to acknowledge the support given by the Chairman and Dean of the Faculty of Pharmacy \& Alternative Medicine, The Islamia University of Bahawalpur, Pakistan.

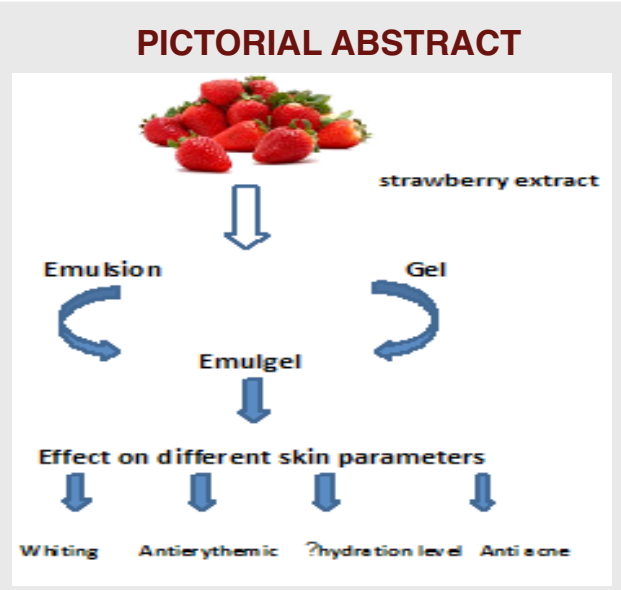

\section{CONFLICTS OF INTEREST}

The authors declare no conflict of interest.

\section{ABBREVIATION USED}

RH: Relative humidity; Q.s: Quantity sufficient; ml: Milliliter; mg: Milligram; g: Gram; rpm: Revolutions per minute.

\section{REFERENCES}

1. Gray J. The word of skin care. Macmillan Press, London. press release. 2000.

2. Heinrich U, Herling M, Binder S, Gerlach N, Tronnier H. Evaluation of the efficacy of skin surface parameters by image analysis with the SELS-method.

3. Jain A, Gautam SP, Gupta Y, Khambete H, Jain S. Development and characterization of ketoconazole emulgel for topical drug delivery. Der pharmacia sinica. 2010;1(3):221-31.

4. Akhtar N, Mehmood A, Khan BA, Mahmood T, Muhammad H, Khan S, et al. Exploring cucumber extract for skin rejuvenation. African Journal of Biotechnology. 2011;10(7):1206-16.

5. Määttä-Riihinen KR, Kamal-Eldin A, Törrönen AR. Identification and quantification of phenolic compounds in berries of Fragaria and Rubus species (family Rosaceae). Journal of Agricultural and Food Chemistry. 2004;52(20):6178-87. https://doi.org/10.1021/jf049450r ; PMid:15453684.

6. Ellis CL, Edirisinghe I, Kappagoda T, Burton-Freeman B. Attenuation of meal-induced inflammatory and thrombotic responses in overweight men and women after 6-week daily strawberry (Fragaria) intake. Journal of atherosclerosis and thrombosis. 2011;18(4):318-27. https://doi.org/10.5551/ jat.6114; PMid:21242652.

7. Codex BP. Pharmaceutical Society of Great Britain. London; 1934.

8. Karou D, Dicko MH, Simpore J, Traore AS. Antioxidant and antibacterial activities of polyphenols from ethnomedicinal plants of Burkina Faso. African Journal of Biotechnology. 2005;4(8):823-8.

9. J S. Encyclopedia of pharmaceutical technology. book. 2004;2:1066-70.

10. P J. skin physiology. 2006;2nd ed. english.

11. Shirani G, Ganesharanee R. Extruded products with Fenugreek (Trigonellafoenum-graecium) chickpea and rice: Physical properties, sensory acceptability and glycaemic index. Journal of food engineering. 2009;90(1):44-52. https://doi.org/10.1016/j.jfoodeng.2008.06.004.

12. Dobrev $\mathrm{H}$. Clinical and instrumental study of the efficacy of a new sebum control cream. Journal of cosmetic dermatology. 2007;6(2):113-8. https://doi. org/10.1111/j.1473-2165.2007.00306.x; PMid:17524128.

\section{SUMMARY}

- Strawberry fruit has good antioxidant activity also exhibits beneficial effects on skin. In this investigational study the emulgel loaded with strawberry extract $(4 \%)$ significantly reduced melanin and sebum of the skin. Significantly improved the hydration level of skin by increasing moisture contents. Strawberry emulgel also has good anti-erythemic effects on skin.

Cite this article: Kausar R, Akhtar N. Formulation of an emulgel containing strawberry fruit extract and in-vivo evaluation for different skin parameter. Indian J of Pharmaceutical Education and Research. 2017;51(4):679-83. 